\title{
Enhancement of methodology for protection of structures in contradiction of thermal effects
}

\author{
Elena Rojkova ${ }^{*}$, Nodira Ruzieva, and Zuxritdin Ergashev \\ ${ }^{1}$ Tashkent State Transport, Tashkent, Uzbekistan
}

\begin{abstract}
The research paper is devoted to protection of structures against heat and temperature effects. The necessity of improving the calculation of multilayered fence structures is shown. The solution of a one-dimensional unsteady heat conduction equation with constant and variable coefficients allowing to use of inhomogeneous and anisotropic materials as the fence material is given. An example of the solution of a fence made of inhomogeneous and anisotropic material is given. Solution of heat conduction equation is obtained by the recurrence-operator method. The solution of one-dimensional unsteady heat conduction equation with variable coefficients is obtained using the recurrence-operator method. The possibility of using the solution of the equation for multilayered inhomogeneous anisotropic fence materials is indicated.
\end{abstract}

\section{Introduction}

The object of investigation is the protection of structures from heat influence (heat and cold) by means of bone up of the process of heat flow and correspondent temperature through covering thickness of outer parts, consisting of layers of different thermal protective materials.

In construction thermal physics, such problems are investigated mainly by solving a one-dimensional nonstationary nonlinear (more precisely quasi-linear) heat conduction equation. In general, these characteristics depend on the material properties, its temperature, humidity and can be a given function of the envelope thickness within each envelope layer in the case of continuously heterogeneous material.

Due to the great difficulties in solving the heat transfer equation, engineering calculations are limited to an equation with constant coefficients, for the solution of which the method of separation of variables (Fourier method) is applied. As a result, the solution is obtained in the form of stationary, "standing" heat waves, and the solution itself is expressed in terms of complex and hyperbolic functions [1].

Note the monograph [2] where the Lommel transformation transforms the Bessel differential equations into equations with variable coefficients. Partial cases of this transformation allow us to solve the heat conduction equation in the Bessel functions of the complex argument with various laws of variation of the variable coefficients. In this case, such solutions can be used that are expressed in terms of the constructed functions. By

*Corresponding author: rojkova-elena@mail.ru 
separating the real and imaginary parts, it is possible to obtain solutions in elementary functions in some particular cases. The cases when the heat transfer coefficient is variable and the heat capacity coefficient is constant are discussed in detail, and it is pointed out that it is in principle possible to obtain solutions when not only the heat transfer coefficient $\lambda$ but also the specific heat capacity coefficient changes $c$.

The purpose of this paper is to develop a simpler algorithm for solving the heat conduction equation when both coefficients are variable, using the recurrence-operated method.

\section{Materials and Methods}

In building thermal physics research of the transfer of heat flows through material layers of thickness of enclosure parts of constructions (external walls, floors) and change of temperature in time and on the thickness of enclosure is investigated mainly on a solution of the one-dimensional nonlinear equation of heat conduction which in the general case has a form

$$
c(x) \frac{\partial T}{\partial t}=\frac{\partial}{\partial x}\left[\lambda(x) \frac{\partial T}{\partial x}\right]
$$

Here $T$ is temperature, $t$ is time, $c(x)=c_{0} \rho(x), \lambda(x)$ are given values of coefficients of heat capacity and heat conductivity of individual material layers in the structure, $c_{0}$ is specific heat capacity, $\rho$ is density.

The solution of equation (1) is accompanied by some difficulties, so it is limited to an equation with constant coefficients, which is usually solved by the split-variable method (Fourier method).

$$
c \frac{\partial T}{\partial t}=\lambda \frac{\partial^{2} T}{\partial x^{2}}
$$

The recurrence-operated method is applied to solve the heat conduction equation (1) when coefficients are variable [3].

Let's represent equation (1) in expanded form

$$
-c(x) \frac{\partial T}{\partial t}+\frac{\partial \lambda(x)}{\partial x} \frac{\partial T}{\partial x}+\lambda(x) \frac{\partial^{2} T}{\partial x^{2}}=0
$$

or in compact notation

$$
\lambda \partial_{x}^{2} T+\partial_{x} \lambda \partial_{x} T-c \partial_{t} T=0
$$

where $\lambda, c$ is function of a variable $x ; T$ is function of variables $x, t$; $\partial_{s}^{k}(f)=\partial^{k} f / \partial s^{k}$ ( $k$--th derivative of the function $f$ on the variable $s$ ). Let's convert to the above equation by dividing equation (3) by the coefficient at the major derivative of the allocated variable. In this case, isolating the variable $x$, we obtain: 


$$
\partial_{x}^{2} T+\frac{1}{\lambda} \partial_{x} \lambda \partial_{x} T-\frac{c}{\lambda} \partial_{t} T=0
$$

We will search for the solution of equation (4) as a functional series with undefined functions $Q_{i}^{r}(x) ; r=0,1$ :

$$
T_{r}(x, t)=\sum_{i=0}^{\infty} Q_{i}^{r}(x) \partial_{t}^{i} g(t)=Q_{0}^{r}(x) g+Q_{1}^{r}(x) \partial_{t}^{1} g+Q_{2}^{r}(x) \partial_{t}^{2} g+\cdots
$$

Substituting (5) into (4), we obtain

$$
\begin{gathered}
\frac{c}{\lambda}\left[Q_{0}^{r}(x) \partial_{t} g+Q_{1}^{r}(x) \partial_{t}^{2} g+Q_{2}^{r}(x) \partial_{t}^{3} g+\cdots\right]+ \\
+\frac{\partial_{x} \lambda}{\lambda}\left[\partial_{x} Q_{0}^{r}(x) g+\partial_{x} Q_{1}^{r}(x) \partial_{t} g+\partial_{x} Q_{2}^{r}(x) \partial_{t}^{2} g+\cdots\right]+ \\
\quad+\left[\partial_{x}^{2} Q_{0}^{r}(x) g+\partial_{x}^{2} Q_{1}^{r}(x) \partial_{t} g+\partial_{x}^{2} Q_{2}^{r}(x) \partial_{t}^{2} g+\cdots\right]=0
\end{gathered}
$$

Given similar terms, we get

$$
\left[\left(\partial_{x}^{2}+\frac{\partial_{x} \lambda}{\lambda} \partial_{x}\right) Q_{0}^{r}\right] g+\left[\left(\partial_{x}^{2}+\frac{\partial_{x} \lambda}{\lambda} \partial_{x}\right) Q_{1}^{r}+\frac{c}{\lambda} Q_{0}^{r}\right] \partial_{t} g+\cdots=0
$$

In order for the above equation to hold at all values $x, t$, the expressions in the square brackets must be equated to zero, and the sequence of differential equations must be solved

$$
\begin{aligned}
& \left(\partial_{x}^{2}+\lambda^{*} \partial_{x}\right) Q_{0}^{r}(x)=0 ; \quad\left(\partial_{x}^{2}+\lambda^{*} \partial_{x}\right) Q_{1}^{r}(x)=c^{*} Q_{0}^{r}(x) ; \\
& \left(\partial_{x}^{2}+\lambda^{*} \partial_{x}\right) Q_{2}^{r}(x)=c^{*} Q_{1}^{r}(x) ; \quad \cdots \quad\left(\partial_{x}^{2}+\lambda^{*} \partial_{x}\right) Q_{i}^{r}(x)=c^{*} Q_{i-1}^{r}(x) ; \\
& \lambda^{*}=\partial_{x} \lambda / \lambda ; \quad c^{*}=c / \lambda
\end{aligned}
$$

To solve the first homogeneous equation, we represent it as:

$$
\partial_{x}\left(\partial_{x}+\lambda^{*}\right) Q_{0}^{r}=0
$$

Then the solutions of this equation are the functions $Q_{0}^{0}=1$;

$$
Q_{0}^{1}(x)=\int_{0}^{x}\left(\int_{0}^{x}-\lambda^{*} d x\right) d x
$$

representing initial conditions for determination of other functions by recurrence formulas (6).

Example. Let's suppose that thermal conductivity and heat capacity coefficients are approximated with sufficient accuracy for a given fencing material by linear functions 
$\lambda^{*}(x)=a x+b ; \quad c^{*}(x)=e x+h$, where the constants $a, b, e, h$ are determined experimentally. We are to determine the temperature conditions of the fencing. Let's determine the initial conditions by formulas (7)

$$
Q_{0}^{0}=1 ; \quad Q_{0}^{1}=-\int_{0}^{x} \int_{0}^{x}(a x+b) d x d x=\int\left(a \frac{x^{2}}{2}+b x\right) d x=a \frac{x^{3}}{6}+b \frac{x^{2}}{2}
$$

To determine the function $Q_{1}^{0}$, we write out the second recurrence relation from (6) at $r=0$ and taking into account (7):

$\left(\partial_{x}^{2}+\partial_{x} \lambda^{*}\right) Q_{1}^{0}(x)=c^{*} \times 1 \quad$ and at first, solve the homogeneous equation without the right side $\left(\partial_{x}^{2}+\partial_{x} \lambda^{*}\right) Q_{1}^{0}(x)=0$. Transferring the known function to the right side and integrating the resulting expression twice, we obtain the solution of the homogeneous equation

$$
Q_{1 \text { oдम }}^{0}=-\int_{0}^{x} \int_{0}^{x} \partial_{x} \lambda^{*} d x d x
$$

We turn to the solution of the inhomogeneous equation $\left(\partial_{x}^{2}+\partial_{x} \lambda^{*}\right) Q_{1}^{0}(x)=c^{*}$. Also there, by transferring the known function to the right side, we find a partial solution of the inhomogeneous equation by double integration of the obtained expression

$$
\tilde{Q}_{1}^{0}=\int_{0}^{x} \int_{0}^{x}\left(c^{*}-\partial_{x} \lambda^{*}\right) d x d x
$$

The total solution is the sum of partial solutions $Q_{1}^{0}=C Q_{1 \text { одн }}^{0}+\widetilde{Q}_{1}^{0}$, although here, it is sufficient to know only the second partial solution of the inhomogeneous equation.

The function $Q_{1}^{1}$ is defined by the same recurrence formulas and in the same sequence only instead of $r=0$ one should put $r=1$ and take the second initial condition (8). For practical purposes, it is sufficient to limit ourselves to a small number of approximations and simple approximating elementary functions.

The heat flux $q(t, x)$ is obtained by differentiating the temperature $T(t, x)$ over time $t$.

\section{Results and discussion}

The obtained results can be generalized to multilayer enclosures with the addition of initial conditions and boundary conditions (contact conditions of heat exchange between the layers). It is possible to additionally compose the conditions for assigning such properties of the fence material, which ensure the absence of reflected heat waves and the impossibility of detection by thermal radar units.

The obtained results differ from the known ones in that the solutions are obtained in real rather than complex a function, which does not require additional auxiliary tables. 
In addition, the solutions are obtained in a general form, which makes it possible to conduct a numerical analysis of the optimal variant of the number and dimensions of the layers and the corresponding thermophysical properties of the enclosure material.

\section{Conclusions}

1. In dissimilarity to the existing theory of thermal stability created by O.E. Vlasov and S.I. Muromov, in which calculation using hyperbolic functions of complex variables is considered the only convenient; this paper proposes to use the solution obtained by the recurrence-operated method in real functions. This eliminates the need to use complex calculations using complex numbers, tables, and graphs.

2. The found solution is generalized to the calculation of multilayer enclosures with the formation of additional thermal contact conditions at the boundaries of the layers.

3. The solution achieved in general form allows conducting further research on the choice of the number, thickness, and thermophysical aspects of the material of the layers.

\section{References}

1. Bogoslovsky V.N. Construction thermophysics, p. 415, Moscow High School, (1982)

2. Korenev B.G. Problems of the Theory of Heat Conductivity and Thermoelasticity p. 400, Moscow Science, (1980)

3. Spivakov Y.L. Special classes of solutions of linear differential equations and their applications to anisotropic and inhomogeneous theory of elasticity, p. 186, Tashkent, (1986)

4. Fokin K.F. Construction Heat Engineering of Building Enclosure Parts of Buildings, p. 252, MOSCOW, (2006)

5. Frolov V.N. Special classes of functions in anisotropic theory of elasticity, p. 224 Tashkent, (1981)

6. Rozhkova E.V. On solutions of the problem in stresses with use of Maxwell stress functions Mech Solids 44, pp. 526-536 (2009), doi: 10.3103/S0025654409040049

7. Rozhkova E.V. Recursive-operator Method in Vibration Problems for Rod Systems Mech Solids 44, pp. 915-926 (2010), doi: 10.3103/S0025654409060090

8. Rozhkova E.V. To the Investigation of Plane Wave Propagation in an Elastic Anisotropic Media by a Recursive Operator Method, Mech Solids, 53 pp. 33-44 (2018), doi: 10.3103/S0025654418010041

9. Rozhkova E.V. Solution of the Generalized Oscillation Equation of Rods by a Recursive Operator Method Mech Solids 53, pp.552-567 (2019), doi:10.3103/S0025654418080095

10. Rozhkova E.V. Solutions using the Recursive Operator Method for the Dynamic Equation of Heart and Mass Transfer in Hardening Concrete under Heat Treatment in a Heating Chamber J Appl Mech Tech Phy, 51, pp. 683-692 (2010) doi: 10.1007/S10808-010-0088-9

11. Ergashev Z.Z. Methodology for determining bearing cacasity of subgrade of sand dunes under vibrodynamic effects, International Journal ofScientific and Technology Research pp. 1873-1876 (2019)

12. Vlasov O.Ye. Teplotekhnicheskiy raschet ograzhdayushchikh konstruktsiy, p. 46, Moscow (1933)

13. Muromov S.I. Raschetnyye temperatury naruzhnogo vozdukha i teploustoychivost' ograzhdeniy. Moscow p. 72 (1939) 
14. Slee B, Parkinson T, Hyde R. Can you have too much thermal mass Cutting Edge in Architectural Science, Proc. of the 47th International Conference of the Architectural Science Association, pp. 325-333, (2013)

15. Wang L.S. A study of building envelope and thermal mass requirements for achieving thermal autonomy in an office building, Energy and Buildings, 78. pp. 79-88, (2014)

16. Ellerbrok C. Potentials of demand side management using heat pumps with building mass as a thermal storage, Energy Procedia, 46, pp. 214-219, (2014)

17. Malyavina E.G. Joint calculation of the nonstationary thermal mode of a building and its water heating system. Materials and Technologies in Construction and Architecture, Proc. of Science Forum, 931, pp. 905-909, (2018) 